\title{
Fatigue craze initiation in polycarbonate: study by small-angle $X$-ray scattering
}

\author{
Hristo A. Hristov and Albert F. Yee* \\ Department of Materials Science and Engineering, University of Michigan, Ann Arbor, MI \\ 48109, USA
}

\author{
Li Xie and David W. Gidley \\ Department of Physics, University of Michigan, Ann Arbor, MI 48109, USA \\ (Received 23 November 1992; revised 16 January 1994)
}

\begin{abstract}
The structural changes that occur during the incubation stage of tensile fatigue failure of glassy polycarbonate are investigated by means of small-angle X-ray scattering. The results are interpreted as the formation of voids, or 'proto-crazes', with initial dimensions of $\sim 60 \mathrm{~nm}$ and $150-200 \mathrm{~nm}$ in directions parallel and perpendicular to the load axis, respectively. These values are in the range expected for a void in a static equilibrium, and practically coincide with the initial void dimensions measured in glassy polymers stretched under monotonic loading.
\end{abstract}

(Keywords: fatigue craze initiation; polycarbonate; small-angle X-ray scattering)

\section{INTRODUCTION}

Fatigue in polymers has been a subject of considerable interest during the past two decades. It has been established that, under a variety of experimental conditions, the fatigue failure develops in two phases ${ }^{1}$ - the initiation phase, in which crazes and/or microcracks form, followed by the propagation phase, in which cracks grow (continuously or discontinuously) until final rupture. The propagation phase has been extensively studied in various polymers, blends and composites ${ }^{1-5}$. It seems that the basic phenomena are well described, and there is a fair amount of information with regard to the factors influencing the occurrence of various processes in this phase $^{1.5}$. In contrast, the knowledge with regard to initiation of structural inhomogeneities during fatigue ${ }^{1}$, and static loading as well ${ }^{6}$, is still very incomplete. While the crack propagation phase is undoubtedly important, there are important reasons why the initiation phase must also be studied. In many materials, including polymers, the initiation phase is a significant or even majority fraction of the total fatigue lifetime to failure. It is therefore useful to be able to predict and/or to influence the lifetime in this phase. This is especially so in those situations where the occurrence of any crack leads to functional failure even though the material may still possess structural strength. Finally, the fundamental question of how an ostensibly homogeneous material incubates a craze is inherently interesting.

In thermoplastics, crazing is always observed to precede cracking. Thus crack incubation is synonymous with craze initiation. It is reasonable to hypothesize that craze initiation or nucleation begins with the growth of

* To whom correspondence should be addressed voids whose dimensions are significantly larger than the size of the 'frozen-in' density fluctuations, known to exist in disordered materials. The existence of such nuclei was proposed by Argon et al. ${ }^{7}$. Mechanical properties studies in our laboratory show that the fracture toughness of a polymer glass is reduced by cyclic loading ${ }^{22}$. This observation is also consistent with a change in the spectrum of inherent flaws in the glass, and perhaps the growth of significant voids.

There are several microscopic models describing craze nucleation under static load ${ }^{7-10}$, but there is no universally accepted theory. From the viewpoint of the present work, it is important to note that the above models postulate in some form the emergence of a critical size nucleus (craze). It has been estimated ${ }^{6}$ that the lateral critical (initial) craze size in amorphous, drawn polystyrene (PS) is of the order of $75 \mathrm{~nm}$. In drawn poly(methyl methacrylate) (PMMA) and polyester, the initial dimensions of microvoids in directions parallel and perpendicular to the draw direction have been measured by SAXS $^{3}$. The initial void dimensions in various glassy polymers are typically in the ranges $70-90 \mathrm{~nm}$ and $190-200 \mathrm{~nm}$, in the two directions respectively ${ }^{3}$. In semicrystalline, highly oriented materials the respective void sizes appear to be an order of magnitude smaller $(5-20 \mathrm{~nm} \text { and } 9-32 \mathrm{~nm})^{3}$. The dimensions of fatigueinduced voids in semicrystalline polyoxymethylene (POM) practically coincide with those created by static loading ${ }^{11}$. The structure of 'mature' crazes obtained after cyclic loading has been investigated by various methods ${ }^{1,5}$. In situ observations of the microstructural changes during cyclic bending at high amplitudes have been reported for $\mathrm{PS}^{12}$. To the best of our knowledge, data on the critical precraze void size in conditions of cyclic loading of 
amorphous polymers have not been reported to date. The main goal of the present work is to obtain information with regard to the initial craze dimensions in fatigued, amorphous polycarbonate (PC). This work is a continuation of the investigations performed in our laboratory ${ }^{13,14}$. In our previous work positron annihilation lifetime spectroscopy (PALS) was used to detect the formation of 'holes' up to $2 \mathrm{~nm}$ in size. While the results were rather complex, one possible interpretation is coalescence of smaller voids into large ones upon fatigue $^{14}$. Since the size resolution of the SAXS method is from 2-3 to $200-300 \mathrm{~nm}$, it has the potential of detecting craze nuclei larger than those measured by PALS.

\section{EXPERIMENTAL}

\section{Materials and sample preparation}

The samples used in this work were prepared from compression-moulded polycarbonate resin supplied by the General Electric Co. The moulding conditions have been described previously ${ }^{13,14}$. The smooth dumbbellshaped specimens with thickness $3 \mathrm{~mm}$ were cut at room temperature and the edges were polished manually. To remove the stresses induced by the cutting procedure, the test samples were annealed at $160^{\circ} \mathrm{C}$ for $40 \mathrm{~min}$ $\left(T_{\mathrm{g}} \sim 155^{\circ} \mathrm{C}\right)$ and cooled to room temperature at cooling rate $500^{\circ} \mathrm{C} \mathrm{min}^{-1}$. Prior to the cyclic loading the specimens were aged for $24 \mathrm{~h}$ at $130^{\circ} \mathrm{C}$. The fatigue procedures were performed on a computer-controlled servohydraulic machine (Instron, model 1332), at room temperature, under a constant stress amplitude of $30 \mathrm{MPa}\left(\sigma_{\min } / \sigma_{\max }=0.05\right.$; sinusoidal $)$ and frequency of $5 \mathrm{~Hz}$. The maximum stress amplitude is approximately half of the yield stress in monotonic loading. More detail on the materials, thermal treatment and instrumentation can be found in refs. 13 and 14.

Since at this load amplitude the samples break after 9000-10000 cycles, a set of specimens was prepared by stopping the fatigue process after $1000,3000,5000$ and 7000 cycles (samples $1 \mathrm{~K}, 3 \mathrm{~K}, 5 \mathrm{~K}$ and $7 \mathrm{~K}$ ) respectively. Other specimens deformed up to $55 \mathrm{MPa}$ under static loading (creep craze initiation; sample $0 \mathrm{~K}$ ) and undeformed specimens were used as reference samples for comparison. The fatigue specimens were either unloaded prior to the SAXS measurements, or kept under constant strain. This was accomplished by clamping a steel jig tightly around the loaded specimen before its removal from the Instron machine.

\section{SAXS instrumentation and data analysis}

SAXS measurements were performed by using a Kratky-type camera (Anton Paar, Austria) in $\mathrm{Fe} \mathrm{K}_{\alpha}$ radiation, which was collimated with an entrance slit of $30 \mu \mathrm{m}$. The sample-to-detector distance was $370 \mathrm{~mm}$. The diffracted radiation was registered by a positionsensitive proportional counter (M-Broun, Germany, model OED 50) in $2 \theta$ interval from $0.04-0.06^{\circ}$ up to $6.6^{\circ}$ simultaneously (the minimum accessible angle depends on the geometry and the scattering power of the samples). This angular interval corresponds to a reciprocal space interval from $k_{\min }=0.02-0.03 \mathrm{~nm}^{-1}$ to $k_{\max }=3.3 \mathrm{~nm}^{-1}$, where $|\boldsymbol{k}|=4 \pi \sin \theta / \lambda$ is the usual diffraction vector, $\theta$ is the diffraction angle and $\lambda$ is the wavelength of the radiation $(0.194 \mathrm{~nm})$. The measurements were made on samples both under constant strain and unloaded, and in two diffraction geometries- equatorial (slit parallel to the load axis) and meridional (slit perpendicular to the load axis). The sample strain was chosen to correspond to an initial stress of $30 \mathrm{MPa}$. Since the stress relaxation of the polycarbonate at room temperature is a relatively slow $(\sim 4 \%$ in $24 \mathrm{~h}$ at this stress level) process, it can be assumed that the SAXS measurements were performed at a constant stress. The diffraction curves were obtained from several areas of the samples by moving it in a plane perpendicular to the primary beam. The experimental intensities were corrected for air, background and wide-angle 'parasitic' scattering by using an undeformed sample. The corrected results were extrapolated to zero by using the last few points from the range $2 \theta=0.04-0.06^{\circ}$. It can be estimated that the maximum particle size resolution of the instrument is in the range $150-200 \mathrm{~nm}$. The resulting spectra were further smoothed by Fourier filtering and desmeared (infinitely long slit approximation ${ }^{15}$ ). As will be discussed below, the diffraction results indicate that the shapes of the scattering entities can be approximated by an ellipsoid. It is elementary to show that the desmearing procedures for spheres and ellipsoids are fundamentally equivalent.

A simple method of interpreting the SAXS curves (smeared and desmeared as well), obtained from independently scattering particles, is the Guinier approximation ${ }^{16}$ :

$$
I(k) \sim \exp \left(-k^{2} R_{\mathrm{g}}^{2} / 3\right)
$$

where $I(k)$ is the diffracted intensity and $R_{\mathrm{g}}$ is the radius of gyration. For spherical scatterers (particles or voids) the geometrical radius is given by $R=\sqrt{ }(5 / 3) R_{\mathrm{g}}$. The Guinier approximation gives quantitatively significant results for systems containing monodisperse or narrow size-distribution scatterers. For a polydisperse system the results from the Guinier approximation should be regarded as qualitative. In such cases the most complete and rigorous information is provided by the distance distribution function $(D D F)$ method $^{17}$. It has been shown that, in the case of independent scattering from particles of the same shape, the total intensity is given by ${ }^{17}$ :

$$
I(k)=\int_{0}^{\infty} \rho(a) I_{a}(k) \mathrm{d} a
$$

where $I_{a}(k)$ is the scattering from a particle with characteristic dimension $a$ and $\rho(a)$ is the distance distribution function.

For a large variety of particle shapes, the relationship between the diffracted intensity and $D D F$ has the general form:

$$
k^{n} I(k)=\int k J_{v}^{2}(k a) a \rho(a) a^{m} \mathrm{~d} a
$$

where $J_{v}(x)$ is a $v$ th-order Bessel function of the first kind and the parameters $n, m$ and $v$ depend on the shapes of the scattering entities and the geometry of the experiment.

Equation (3) is a special case of the Titchmarsh transformation, and its solution can be expressed as:

$$
a^{m} \rho(a)=\int_{0}^{\infty}\left[I(k) k^{n}-C_{n}\right] \phi(k a) \mathrm{d} k
$$

where

$$
C_{n}=\lim _{k \rightarrow \infty}\left[k^{n} I(k)\right] \quad \phi(x)=2 \pi \frac{\mathrm{d}}{\mathrm{d} x}\left[x J_{v}(x) N_{v}(x)\right]
$$


Fatigue craze initiation in PC by SAXS: H. A. Hristov et al.

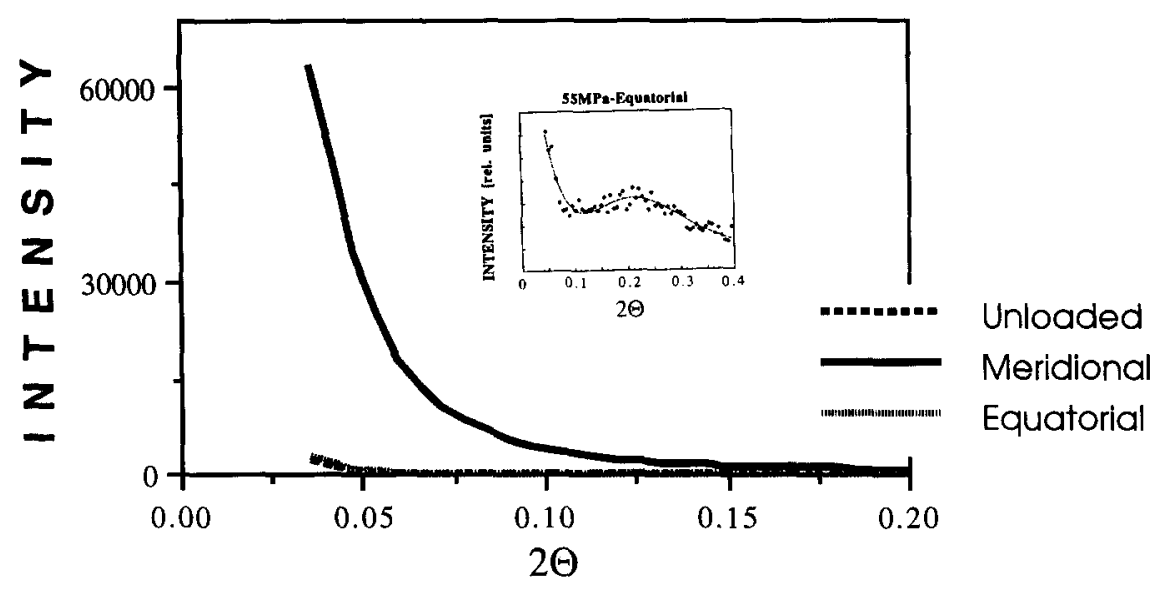

Figure 1 SAXS intensity scans (no corrections) of a sample deformed up to $55 \mathrm{MPa}$ under static loading (sample $0 \mathrm{~K}$ ). The inset shows the equatorial curve from the loaded specimen, corrected for background and wide-angle scattering

and $N_{v}(x)$ is a $v$ th-order Bessel function of the second kind (Neumann function) ${ }^{17}$.

Depending on the shape of the scatterers, the functional form of the kernel $\phi(x)$ and equation (4), respectively, can be rather complicated. For particles with special symmetries, however, as a rule (4) reduces to more tractable forms. As an example the $D D F$ from thin platelets reduces to a cosine Fourier transformation of the function $f(k)=I(k) k^{4}-C_{4}$ (refs. 17, 18):

$$
\rho(t)=-\int_{0}^{\infty} f(k) \cos (k t) \mathrm{d} k
$$

where $I(k)$ is the desmeared intensity and $t$ is the platelet thickness. This approach has been used to obtain the thickness distribution of plate-like voids in charcoals ${ }^{18}$.

\section{RESULTS AND DISCUSSION}

The samples fatigued up to $5 \mathrm{~K}$ (cycles) and $7 \mathrm{~K}$ showed the presence of visible crazes and small cracks, while in samples $1 \mathrm{~K}$ and $3 \mathrm{~K}$ no cracks or crazes were detected by optical microscopy up to a magnification of $60 \times$. The crazes in samples $5 \mathrm{~K}$ and $7 \mathrm{~K}$ were distributed unevenly over the active area $\left(12 \times 9 \mathrm{~mm}^{2}\right)$ of the specimens. Sample $0 \mathrm{~K}$ (deformed to $55 \mathrm{MPa}$ ) is characterized by the presence of a large number of crazes/microcracks, with relatively homogeneous spatial distribution. Optical observation revealed that the thickness of the crazes in the loaded specimens $0 \mathrm{~K}, 5 \mathrm{~K}$ and $7 \mathrm{~K}$ can be as large as $1-2 \mu \mathrm{m}$, while their length was usually an order of magnitude larger. In Figure $I$ are shown the uncorrected equatorial and meridional intensity distributions of sample $0 \mathrm{~K}$ in loaded and unloaded conditions. Since the meridional curve was an order of magnitude more intense than the other curves, in the inset is shown the equatorial curve corrected for background and wide-angle scattering. The diffraction curve from the unloaded specimen practically coincides with the curve from the undeformed specimen (not shown here), which is probably due to the collapse of crazes upon unloading. Similar results have been reported for crazes in PS $^{3}$. From the broad maximum of the equatorial scan, one can obtain an 'interfibrillar' spacing in the range $40-50 \mathrm{~nm}$, which is commonly found for crazes in $\mathbf{P C}^{\mathbf{1 9}, 20}$. The strong diffraction in the meridional direction has been termed 'anomalous scat- tering'21. According to the analysis in the latter work, the meridional scattering is due to: (a) diffraction from the craze itself (slit scattering) with a density difference from the bulk $\rho\left(1-V_{\mathrm{f}}\right)$, where $V_{\mathrm{f}}$ is the volume fraction of the craze fibrils, and (b) complete internal reflection from the craze-bulk interface. The meridional curves from crazes in PS have been analysed also as a reflection from partially smooth surfaces ${ }^{23,24}$. From the analysis in these works, one can conclude that in order to obtain significant reflection the crazes in the material under investigation must fulfil three conditions simultaneously: (i) existence of a sufficiently large planar interface (this condition is usually fulfilled in the case of large 'mature' crazes); (ii) sufficiently smooth interface (depends on the material and the external variables such as temperature, sample thickness, environment and others); and (iii) the interface must be properly oriented with respect to the incoming X-ray beam. It is reasonable that in samples containing a large number of mature crazes these conditions can be satisfied (at least partially); however, the same is not clear in the case of small short crazes and precraze formations in bulk PC. The amount of the reflected intensity depends on a number of $a$ priori unknown factors ${ }^{23,24}$, and it is useful to estimate experimentally the maximum possible contribution from reflection in our case. To achieve this goal, several samples were fatigued at $30 \mathrm{MPa}$ until fracture, and the sample with the most planar fracture surface was used for the SAXS measurements. Since we are interested in the initiation stage of the fatigue process, the length of the X-ray beam was limited to $3 \mathrm{~mm}$ and the sample was so positioned that predominantly the region around the initiation site of the fracture crack/craze was irradiated. The sample was tilted along the two axes perpendicular to the stress axis until maximum reflected intensity was obtained. In Figure 2 the reflection intensity curve is compared to the meridional curve from sample $0 \mathrm{~K}$. These results show that the angular dependence of the two curves is different and the total reflected intensity is approximately half of that of the meridional curve. Taking into account that in the ideal case both craze surfaces will reflect, one obtains the result that the maximum reflected intensity from a huge craze with total interface area $18 \mathrm{~mm}^{2}$ (the sample thickness is $3 \mathrm{~mm}$ ) will be comparable to the meridional intensity from specimen $0 \mathrm{~K}$. After the SAXS measurements sample $0 \mathrm{~K}$ was cut, 


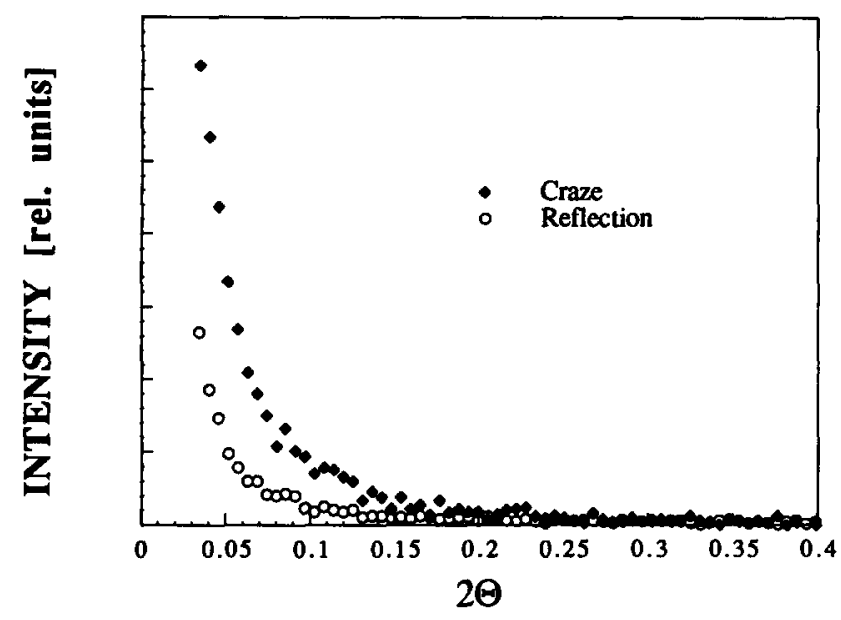

Figure 2 Meridional intensity curve of sample 0K (loaded, corrected for background and wide-angle scattering: $\bullet$ ) and reflection curve from broken specimen (corrected for background, wide-angle scattering and absorption: 0 )

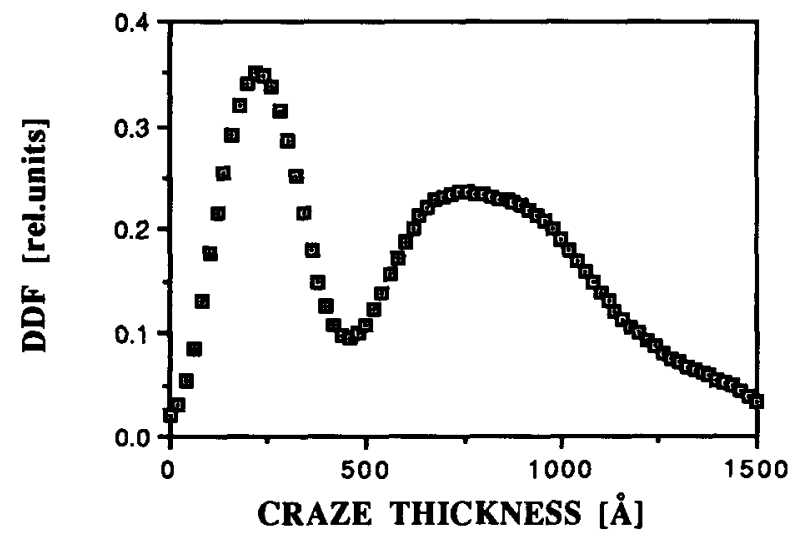

Figure 3 Distance distribution function in meridional direction (craze thickness distribution) of sample $0 \mathrm{~K}$

and the typical craze dimensions (measured by optical microscopy) were found to be $12 \times 4 \mu \mathrm{m}^{2}$. Since the cross-section of the X-ray beam at the sample position is $\sim 7 \mathrm{~mm} \times 50 \mu \mathrm{m}$, we found that no more than five crazes on each side of the sample can be irradiated simultaneously. Now if we assume that all of them are ideally oriented with respect to the incident beam, the maximum interface area available for reflection will be $\sim 2000 \mu \mathrm{m}^{2}$. Thus the maximum reflected intensity from sample $0 \mathrm{~K}$ will be at least four orders of magnitude smaller than that shown in Figure 2. It is impossible to obtain a similar estimate for the reflection component from the fatigued specimens $(1 \mathrm{~K}, 3 \mathrm{~K}, 5 \mathrm{~K}$ and $7 \mathrm{~K})$, but it is obvious that it should be much smaller than that from sample $0 \mathrm{~K}$. The above estimate strongly indicates that the contribution from the craze-bulk interface reflection for the samples $0 \mathrm{~K}, 1 \mathrm{~K}, 3 \mathrm{~K}, 5 \mathrm{~K}$ and $7 \mathrm{~K}$ should be negligible. Another strong indication is the fact that the angular change of the desmeared meridional intensity of all samples follows the $k^{-4}$ law $\left(k^{-3}\right.$ for the smeared one) in accordance with equation (5) and (6), derived under the assumption that volume diffraction is the main scattering process ${ }^{17,18}$. While the reflection phenomenon requires further investigation, it is clear that its contribution can be ignored in the present analysis. Thus the meridional curve in Figure $l$ is due to diffraction from the crazes in the specimen and contains information about their thicknesses. The Guinier plot $\left(\log I\right.$ vs. $\left.k^{2}\right)$ showed considerable curvature at all diffraction angles, which indicates that the Guinier approximation is unreliable for the size determination. Taking into account the fact that the lateral dimensions of the crazes are typically outside the SAXS range, one can use equation (5) to derive the thickness distribution. The cosine transform of the appropriately corrected meridional curve is shown in Figure 3. The value of the constant $C_{4}$ used to compute this curve, as well as all other $D D F$ curves shown below, was obtained from the expression ${ }^{17}$ :

$$
\int_{0}^{\infty}\left[I(k) k^{4}-C_{4}\right] \mathrm{d} k=0
$$

The results in Figure 3 show relatively broad thickness distribution, with the majority of the distances grouped below $50 \mathrm{~nm}$. The distribution is characterized by three broad peaks centred at $\sim 25, \sim 70$ and $\sim 90 \mathrm{~nm}$, respectively, and the last two peaks overlap. The $D D F$ curves obtained from measurements of different portions of sample $0 \mathrm{~K}$ showed some variations of the thickness distribution; however, their general shape is similar to that shown in Figure 2. It should be noted that the termination of the curve at $150 \mathrm{~nm}$ does not mean that crazes with larger dimensions cannot be found. Rather, the larger dimensions are outside the range of the instrument used. The same is true for the curves obtained from the other samples. The $D D F$ curves were tested for termination effect ripples by changing the upper limit of integration in equation (5).

The diffraction curves from the crazed areas of fatigued samples $5 \mathrm{~K}$ and $7 \mathrm{~K}$ are similar to those from sample $0 \mathrm{~K}$. Scanning various areas of the fatigued samples revealed that large regions (especially in specimens $1 \mathrm{~K}$ and $3 \mathrm{~K}$ ) are practically unaffected by the fatigue process-the intensity curves were identical to those from the unfatigued reference sample. The regions of samples $1 \mathrm{~K}$ and $3 \mathrm{~K}$ that do give diffraction different from that of the reference sample were considerably localized.

In Figure 4 are shown the curves obtained from an area with no visible crazes of the loaded sample $7 \mathrm{~K}$ (which has experienced the highest number of cycles), which gives significant diffraction. For comparison we show, in Figure 5 , the diffraction from an analogous portion of the loaded specimens $1 \mathrm{~K}$ (which has experienced the lowest number of cycles). Inspection of Figures 4 and 5 demonstrates that the results from the two specimens are qualitatively similar. The same is true for samples $3 \mathrm{~K}$ and $5 \mathrm{~K}$, when

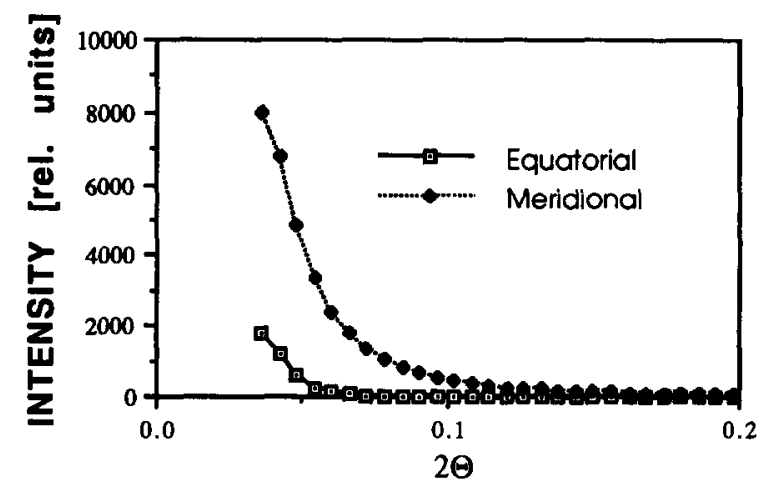

Figure 4 SAXS intensity scans of sample $7 \mathrm{~K}$ (loaded), corrected for air and wide-angle scattering 


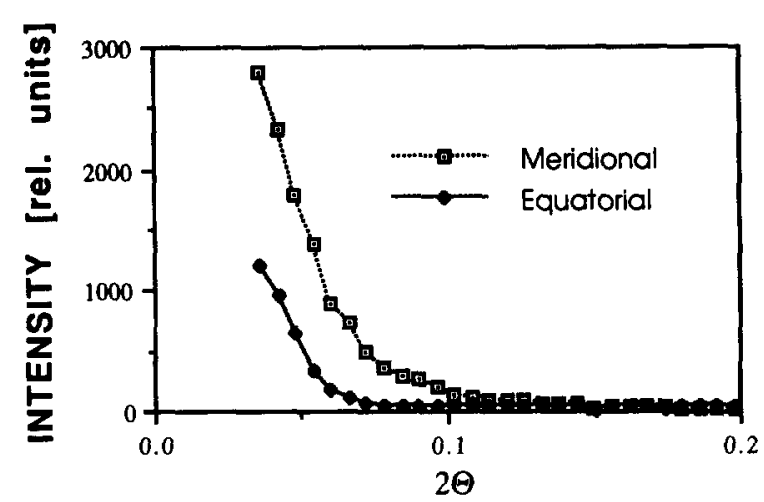

Figure 5 SAXS intensity scans of sample 1K (loaded), corrected for air and wide-angle scattering

the diffraction is obtained from appropriate areas of the specimens. Since the results are similar, the respective curves are not shown separately. The meridional curves in both figures are more intense than the equatorial ones, but the difference is much smaller in comparison to sample 0K (Figure I). The equatorial intensities from the loaded samples deviate from those of the unloaded ones (not shown here) only at very small angles, and the diffraction from the unloaded samples coincides with the scattering curve from the unfatigued reference sample. These results clearly indicate that in some portions of samples $1 \mathrm{~K}$ and $3 \mathrm{~K}$, and in still uncrazed areas of samples $5 \mathrm{~K}$ and $7 \mathrm{~K}$, are formed small numbers of anisotropic voids, which we shall term 'proto-crazes'. The minimum lateral size of the voids was estimated from the Guinier plots of the equatorial intensities, and was found to be in the range $150-200 \mathrm{~nm}$. Similarly to sample $0 \mathrm{~K}$ the Guinier plots $\left(\log I\right.$ vs. $\left.k^{2}\right)$ of the meridional intensities showed considerable curvature at all diffraction angles, which indicates that the Guinier approximation is unreliable for the size determination. To obtain the void thickness distribution, the meridional curves were transformed by using equation (5), and the resulting $D D F$ s are presented in Figure 6. The curve from sample $1 \mathrm{~K}$ demonstrates that the average void thickness is $\sim 60 \mathrm{~nm}$. It seems that this distance is quite typical, since a broad peak at $\sim 60 \mathrm{~nm}$ is present in the curves corresponding to samples $3 \mathrm{~K}$ and $5 \mathrm{~K}$. In addition the curves show development of a second peak at about $120 \mathrm{~nm}$. In sample $7 \mathrm{~K}$ the thickness distribution is reasonably homogeneous in the range $30-130 \mathrm{~nm}$.

Inspection of Figures 3 and 6 indicates that, in the samples with most extensive damage $(0 \mathrm{~K}$ and $7 \mathrm{~K})$, the size distribution in direction parallel to the stress axis is much broader than in the 'low-damage' samples $1 \mathrm{~K}, 3 \mathrm{~K}$ and $5 \mathrm{~K}$. Another significant difference is the very low number or absence of void dimensions lower than $\sim 30 \mathrm{~nm}$ in the latter samples.

Since the aspect ratio of the voids is approximately 3 , the requirement of a very large aspect ratio in order to use equation (5) is not fulfilled exactly. In this situation the question is: What is the systematic error introduced by using equation (5)? An upper estimate can be obtained if one assumes that the voids are of a spherical shape. The $D D F$ for spherical particles can be derived from (4) by using a kernel in the form ${ }^{17}$

$$
\phi(x)=\cos (2 x)\left[1-\left(2 / x^{2}\right)\right]-[2 \sin (2 x) / x]\left[1-\left(2 / x^{2}\right)\right]
$$

The curves computed by using the spherical approxi- mation have the same shapes as those shown in Figure 6 , but the respective maxima are shifted by $\sim 10 \mathrm{~nm}$ towards larger values.

The results reported in this work can be consistently explained if one assumes that at an early stage of the fatigue process ( $\sim 1000$ cycles at $30 \mathrm{MPa}$ amplitude) the structural changes lead to formation of voids or 'protocrazes' with minimum lateral dimensions in the range $150-200 \mathrm{~nm}$ and thickness $\sim 60 \mathrm{~nm}$. It is necessary to point out that, while the shape of the Guinier plots (not shown here) is typical for single widely spaced particles/ voids, aggregation of closely packed, small, irregular voids will diffract as one large void. It is possible that the diffraction curves presented here contain contributions from the two types of voids, namely large single and groups of small, closely packed voids. Since the SAXS method cannot distinguish between the two cases, the sizes reported here mean either the actual sizes of a large single void or the sizes of the envelope containing the smaller voids. The low number of thicknesses below $\sim 40 \mathrm{~nm}$ suggests that void edges are rather blunt, which is an indication that shear flow may be an important factor in the proto-craze formation process. Further development increases the proto-craze dimensions and leads to formation of sharper edges. It has been well established that mature crazes can have a very sharp tip $^{1,6}$. However, very small dimensions have never been detected during the initiation stage of the process. These facts seem to contradict the idea that a craze starts from very small voids $(1-2 \mathrm{~nm})$, which further grow and interconnect ${ }^{6,25}$. On the other hand, it is quite possible that such voids are unstable. Since the hydrostatic tension at the void surface $\left(\sigma_{0}\right)_{\mathrm{s}}$ is inversely proportional to the void diameter, i.e. $\left(\sigma_{0}\right)_{\mathrm{s}}=4 \Gamma / D$, where $\Gamma$ is the surface energy $^{6}$, a large external stress is needed to keep them from closing. If small voids are formed during the non-equilibrium fatigue process, most of them will close subsequently. A small portion of the voids, 'immersed' in a sufficiently large local stress field, will grow rapidly until stable dimensions are reached. The equilibrium dimension can be estimated for the simplistic case of a spherical void subjected to dilatational stress. Taking into account that chain disentanglement rather than chain scission is probably the dominant process in fatigue craze initiation $^{1}$, the value of the surface energy $\Gamma$ should be close to the van der Waals surface energy, i.e. $\Gamma \sim 0.04 \mathrm{~J} \mathrm{~m}^{-2}$ (ref. 6). For external stresses $20-40 \mathrm{MPa}$ $\left(\sigma_{0}\right)_{\mathrm{s}}$ varies from 7 to $14 \mathrm{MPa}$, and one can compute that the void diameter ranges from 120 to $200 \mathrm{~nm}$. While this

Max. Load 30MPa; 1000 - 7000 Cycles

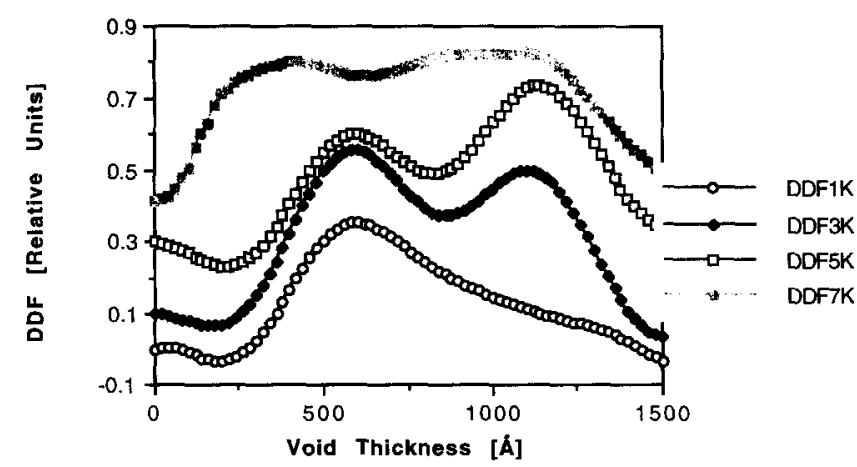

Figure 6 Distance distribution function in meridional direction of samples $1 \mathrm{~K}, 3 \mathrm{~K}, 5 \mathrm{~K}$ and $7 \mathrm{~K}$. The zeros along the vertical axis are shifted 
is a rather crude estimate, it shows that the initial void dimensions measured experimentally in this work are in the expected range. If aggregations of small voids are present in the material, they will be stable under constant external field, despite the fact that a small single void is unstable.

As mentioned above, scanning of various portions of the specimen revealed considerable localization of the craze initiation sites, i.e. they are not distributed homogeneously throughout the sample surface. In this situation it is useful to estimate their concentration range. To this end the diffraction curves from the fatigued PC samples were compared to those obtained from a PC specimen containing a known concentration (10\%) of polystyrene (PS). Since PC-PS are immiscible, the PS phase is in the form of quite uniform spherical particles randomly distributed in the PC matrix. The average size of the PS particles (as derived from SAXS and TEM measurements) was found to be $\sim 100 \mathrm{~nm}$. The diffraction curves from the PC-PS specimens can then be used to calibrate the results from the fatigued samples. The desmeared intensities of the two systems at zero diffraction angle can be written as ${ }^{16}$.

$$
\begin{gathered}
I_{\mathrm{PC}-\mathrm{PS}}(0)=c N_{\mathrm{PS}}\left(\rho_{\mathrm{PC}}-\rho_{\mathrm{PS}}\right)^{2} V_{\mathrm{PS}}^{2} \\
I_{\mathrm{PC}-\text { void }}(0)=c N_{\text {void }}\left(\rho_{\mathrm{PC}}\right)^{2} V_{\text {void }}^{2}
\end{gathered}
$$

where $c$ is an instrumental constant that can be determined from the first equation in (7); $\rho_{\mathrm{PC}}$ and $\rho_{\mathrm{PS}}$ are the electron densities of the two polymers, which can be computed from the macroscopic densities of the respective amorphous and crystalline phases, and the unit-cell dimensions of the PC and PS; $N_{\mathrm{PS}}$ and $N_{\text {void }}$ are the concentrations of the respective scatterers; and $V_{\mathrm{PS}}$ and $V_{\text {void }}$ are the respective volumes. The experimental intensities were extrapolated to zero and by using (7) we found that the void fraction in sample $1 \mathrm{~K}$ varies from 0 to $0.1 \mathrm{vol} \%$, while in sample $7 \mathrm{~K}$ the variation is $0-0.4$ vol\%.

It is customary to determine the beginning of the craze/crack propagation stage by the changes of the optical properties of the specimens ${ }^{1}$. Our measurements demonstrate that the end of the craze initiation phase, when measured by SAXS, is $\sim 1000$ cycles, while the optical methods will give $4000-5000$ cycles. This considerable discrepancy indicates that the term 'craze initiation' needs a more precise definition.

\section{CONCLUSION}

The results described above indicate that craze initiation in amorphous polycarbonate during cyclic loading starts with formation of anisotropic voids, with the short dimension of $\sim 60 \mathrm{~nm}$ along the stress axis, and minimum lateral dimensions $150-200 \mathrm{~nm}$. These values are in the range of values obtained from uniaxially drawn glassy polymers ${ }^{3,6}$. At this stage of the investigation, we are not able to conclude whether this is a coincidence or a manifestation of some fundamental property of polymers under load. However, the results are consistent with our earlier measurements using PALS, which indicate that under fatigue hole coalescence can occur. This may be the origin of formation of the proto-crazes.

\section{ACKNOWLEDGEMENTS}

This work is supported by the Department of Energy under Grant No. DE-FG02-88ER45366. We are indebted to Professor Jerold M. Schultz from the University of Delaware for helpful comments and discussion. We thank Ms Xiaowei Li from the University of Michigan for her help in performing the fatigue experiments.

\section{REFERENCES}

1 Sauer, J. A. and Hara, M. Adv. Polym. Sci. 1990, 91/92, 67

2 Hertzberg, R. W. and Manson, J. A. 'Fatigue of Engineering Plastics', Academic Press, New York, 1980

3 Kusenko, V. S. and Tamuzs, V. P. in 'Fracture Micromechanics of Polymer Materials', Martinus Nijhoff, Boston, 1981

4 Takemori, M. T. Adv. Polym. Sci. 1990, 91/92, 263

5 Doll, W. Adv. Polym. Sci. 1983, 52/53, 105

6 Kramer, E. J. Adv. Polym. Sci. 1983, 52/53, 1

7 Argon, A. and Hannoosh, J. G. Phil. Mag. 1977, 36, 1195

8 Kawagoe, M. and Kitagawa, M. J. Polym. Sci., Polym. Phys. Edn. 1981, 19, 1423

9 Gent, A. N. J. Mater. Sci. $1970,5,925$

10 Kaush, H. H. 'Polymer Fracture', Springer, Berlin, 1987

11 Wendorff, J. H. Prog. Colloid. Polym. Sci. 1979, 66, 135

12 Brown, H. R., Kramer, E. J. and Bubeck, R. A. J. Polym. Sci. Polym. Phys. Edn. 1987, 25, 1765

13 Liu, L. B., Gidley, D. and Yee, A. F. J. Polym. Sci., Polym. Phys. Edn. 1992, 30, 231

14 Liu, L. B., Yee, A. F. and Gidley, D. J. Polym. Sci., Polym Phys. Edn. 1992, 30, 221

15 Glatter, O. in 'Small Angle X-ray Scattering' (Eds. O. Glatter and O. Kratky), Academic Press, New York, 1982, p. 119

16 Porod, G. in 'Small Angle X-ray Scattering' (Eds. O. Glatter and O. Kratky), Academic Press, New York, 1982, p. 17
Fedorova, I. S. and Schmidt, P.J. Appl. Crystallogr. 1978, 11, 405

18 Pringle, O. A. and Schmidt, P. W. J. Colloid Interface Sci. 1977, 60, 252

19 Paredes, E. and Fisher, E. W. Makromol. Chem. 1979, 180, 2707

20 Detenmaier, M. and Leberger, D. Adv. Polym. Sci. 1990, 91/92, 119

21 Brown, H. R. and Kramer, E. J. J. Macromol. Sci.-Phys. (B) 1981, 19, 487

$22 \mathrm{Li}, \mathrm{X}$. and Yee, A. F. unpublished results

23 Westbrook, P. A., Fellers, J. F., Cakmak, M., Lin, J. S. and Hendriks, R. W.J. Polym. Sci., Polym. Phys. Edn., 1983, 21, 1913

24 Tang, M., Fellers, J. F. and Lin, J. S. J. Polym. Sci., Polym. Phys. Edn. 1984, 22, 2215

25 Kambour, R. P. J. Polym. Sci., Macromol. Rev. 1973, 7, 1 